\title{
Press and injection hybrid molding of glass fiber reinforced thermoplastics
}

\author{
K. Tanaka, Y. Fujita \& T. Katayama \\ Department of Biomedical Engineering, Doshisha University, Japan
}

\begin{abstract}
To obtain light-weight vehicles, which is one of the major solutions of the automotive industry to reduce $\mathrm{CO}_{2}$ emissions, the use of fiber reinforced thermoplastic composite material (FRTP) has attracted much attention. For molding methods of FRTP, there are press molding using continuous fiber reinforced thermoplastics and injection molding using short or long fiber reinforced thermoplastics. Although injection molding can mold complex shapes, its mechanical property is not enough for the use as a structural material. On the other hand, press molding using continuous fiber reinforced thermoplastics is excellent in strength and elastic modulus, however, it is difficult to mold three-dimensional complex shapes. The novel molding technology, introduced in this paper, has been developed to gain new perspectives in FRTP by combining press molding and injection molding. This novel hybrid molding technology is suited to mold structural components with high-strength and high-stiffness. Although the interfaces between rib structures with short or long fiber reinforced thermoplastics and the laminated outer shell with the continuous fiber reinforced thermoplastic laminate, the interfacial properties has not been clarified yet. In this study, press and injection hybrid molding that can mold products of complex shapes was introduced, and demonstrator products were molded. To evaluate the bonding strength between rib structures and laminated outer shell of press and injection hybrid molding composites, tensile test of T-shape specimen were conducted.

Keywords: press and injection hybrid molding, composite manufacturing, glass fiber reinforced thermoplastics, bonding strength, rib, FRTP.
\end{abstract}

\section{Introduction}

Lightening vehicle weight to reduce energy consumption and $\mathrm{CO}_{2}$ emissions is one of the major issues for the automotive industry. Since fiber reinforced 
thermoplastic composite material (FRTP) have advantages in high-specific strength, high-specific modulus and recyclability, the demand for FRTP is increasing $[1,2]$. Many dedicated researches to realize the FRTP component parts, such as developments of high-speed molding process of FRTP $[3,4]$ and formability evaluation of CFRTP [5] etc., have been carried out.

There are various structural requirements, such as complex geometries, large projected area, and thin parts, to be used in the automotive industry. Molding methods of FRTP are mainly divided into the following two methods: one is "press molding" that employs continuous fiber reinforced thermoplastic laminates, and the other is "injection molding" that employs short or long fiber reinforced thermoplastics. Although injection molding can mold complex shapes, its mechanical property is not enough for the use as a structural material because of the short fiber length. On the other hand, press molding using continuous fiber as reinforcement is excellent in strength, elastic modulus and the dimensional accuracy of the molded product, however, it is difficult to mold products of complex shapes.

The novel hybrid molding technology, called "press and injection hybrid molding", is the molding method that combines "press molding" that employs continuous fiber reinforced thermoplastics, and "injection molding" that employs short or long fiber reinforced thermoplastics. This press and injection hybrid molding can mold high strength, high stiffness and complex shaped products. In previous studies, Fiorotto and Lucchetta reported that bending stiffness and strength of FRTP element made by hybrid molding (thermoforming and over-injection molding) [6]. Aurrekoetxea et al. investigated the effects of the processing parameters on the tensile shear strengths of the bonding interface which was generated during overinjection molding/thermoforming hybrid process [7]. Although the interfacial properties between rib structures with short or long fiber-reinforced thermoplastics and the laminated outer shell with the continuous fiber reinforced thermoplastic laminate plays a critical role on the mechanical properties of FRTP, the interface properties have not been clarified yet.

In this study, press and injection hybrid molding that can mold products of complex shapes was introduced, and demonstrator products were molded. To evaluate the bonding strength between rib structures and laminated outer shell of press and injection hybrid molding composites, tensile test of T-shape specimen were conducted.

\section{Materials and experimental procedures}

\subsection{Materials}

The glass fiber reinforced thermoplastic (GFRTP) that consist of continuous glass fiber twill woven fabrics impregnated with a polyamide (PA6) as matrix (TEPEX, Dynalite 102-RG600, Bond Laminates, Germany, Tensile strength: $404 \mathrm{MPa}$, Modulus: $22.4 \mathrm{GPa}$, Melting temperature: $220^{\circ} \mathrm{C}$ ) were used. The thicknesses of the laminates used in this study were 1.0 and $2.0 \mathrm{~mm}$, and 
the fiber volume content was $50 \%$ and the density was $1.78 \mathrm{~g} / \mathrm{cm}^{3}$. The short glass fiber reinforced polyamide 6 (PA6) pellet (1015GC3, UBE, Japan) was used for injection molding. This PA6 pellet has the fiber weight fraction of $15 \%$ (the fiber volume fraction of $7.2 \%$ ) and the average length of the short fibers is $0.7 \mathrm{~mm}$.

\subsection{Press and injection hybrid molding}

The schematic drawing of the press and injection hybrid molding process is shown in Fig. 1. The press and injection hybrid molding process is to combine press molding that employs the continuous fiber composite laminates with injection molding and the rib structures that employ the short fiber reinforced thermoplastic pellet. After the glass fiber reinforced thermoplastic (GFRTP) laminate sheet was heated in a heater at $260^{\circ} \mathrm{C}$ and placed inside the mold, GFRTP laminate was press-molded and short fiber reinforced thermoplastic was injected into space for the ribs. The demonstrator product that was manufactured by the press and injection hybrid molding is shown in Fig. 2. Table 1 shows the parameters for the press and injection hybrid molding. The press molding process was performed at constant press pressure of $25.5 \mathrm{MPa}$ and at holding pressure of $10 \mathrm{MPa}$. To understand the temperature histories of laminated outer shell during molding process, the temperature of the surface which will be attached to the injected rib was measured using thermocouples.

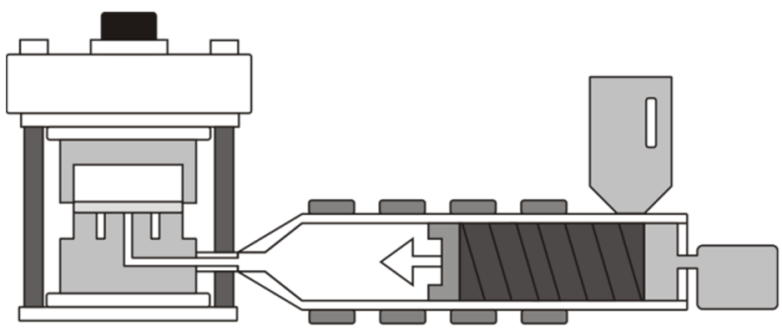

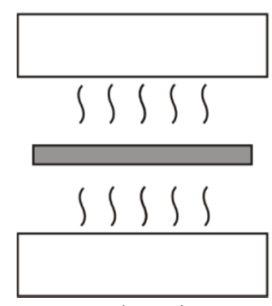

Preheating

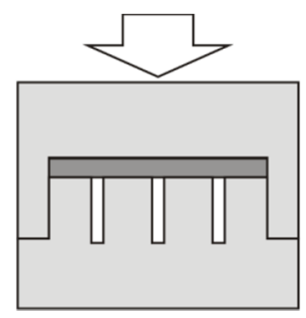

Press molding

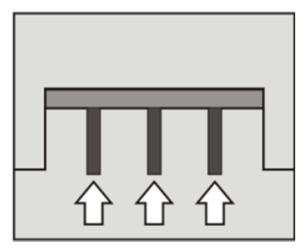

Injection molding

Figure 1: Schematic drawing of the press and injection hybrid molding process. 


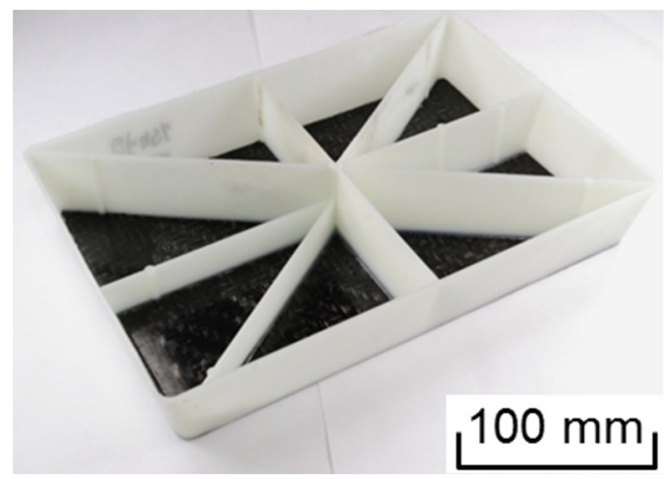

Figure 2: Demonstrator manufactured by the press and injection hybrid molding.

Table 1: Processing parameters of the press and injection hybrid molding.

\begin{tabular}{c|l|c}
\hline \multirow{2}{*}{ Preheating } & Laminate temperature $\left[{ }^{\circ} \mathrm{C}\right]$ & 260 \\
& Heating time $[\mathrm{s}]$ & 240 \\
\hline \multirow{3}{*}{ Press molding } & Pressing pressure $[\mathrm{MPa}]$ & 25.5 \\
& Holding pressure $[\mathrm{MPa}]$ & 10 \\
& Mold temperature $\left[{ }^{\circ} \mathrm{C}\right]$ & 80 \\
\hline \multirow{3}{*}{ Injection molding } & Injection speed $[\mathrm{mm} / \mathrm{s}]$ & 60 \\
& Melt temperature $\left[{ }^{\circ} \mathrm{C}\right]$ & 280 \\
& Cooling time $[\mathrm{s}]$ & 40 \\
\hline
\end{tabular}

\subsection{T shape tensile test}

The bonding strength of the interface between laminated outer shell and rib structure was measured by tensile tests of T shape specimens. T shape tensile test specimens were cut out from the demonstrator products as shown in Fig. 3. The dimensions of the specimen were $15 \mathrm{~mm}$ in width (parallel to rib direction) and $35 \mathrm{~mm}$ in length (orthogonal to rib direction). Aluminum tab was bonded to the laminated outer shell using an epoxy adhesive and the test specimen was fixed to universal material testing machine (5566, Instron, U.S.A.) as shown in Fig. 3. T shape tensile tests were conducted at a cross head speed of $0.017 \mathrm{~mm} / \mathrm{sec}$ and at the room temperature. 

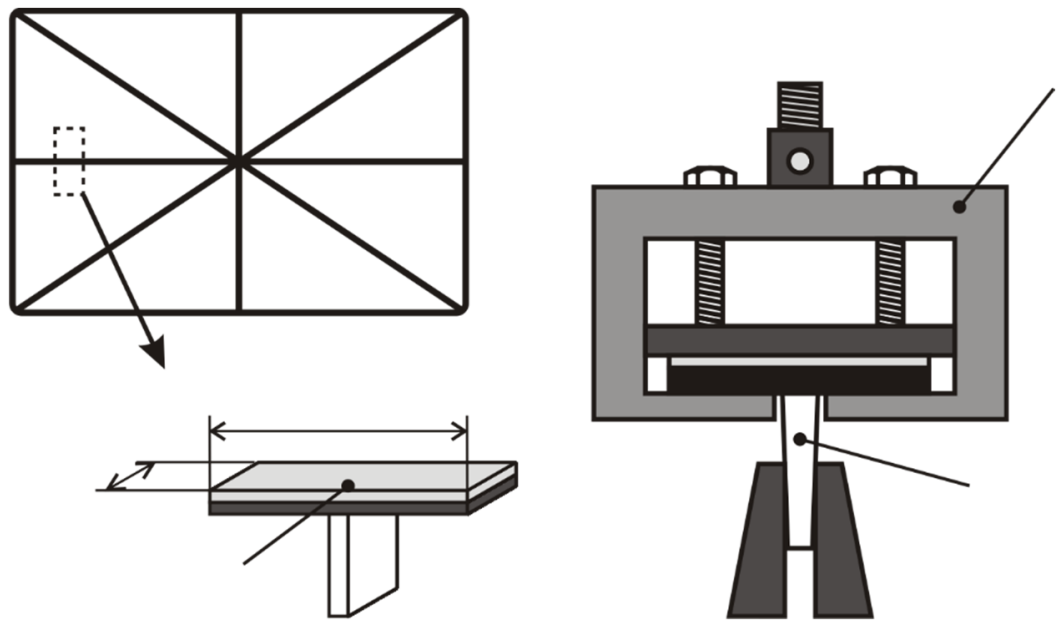

Figure 3: Schematic drawing of T shape specimen and tensile test.

\section{Results and discussions}

The load-displacement curves for T-shape tensile tests for different laminate thicknesses $(1.0 \mathrm{~mm}$ and $2.0 \mathrm{~mm})$ are shown in Fig. 4. There is remarkable difference in load-displacement curves between specimens for thicknesses of 1.0 $\mathrm{mm}$ and $2.0 \mathrm{~mm}$. Fig. 5 shows the magnified cross-sectional view for each test specimen at the interface between rib and laminated outer shell. Comparing the heights that laminated outer shell penetrates into the rib structures, $2.0 \mathrm{~mm}$ thick laminate had larger penetrated height than $1.0 \mathrm{~mm}$ thick laminate. Fig. 6 shows the relationship between the penetrated height and tensile fracture force. Tensile fracture force increased as the penetrated height became large.

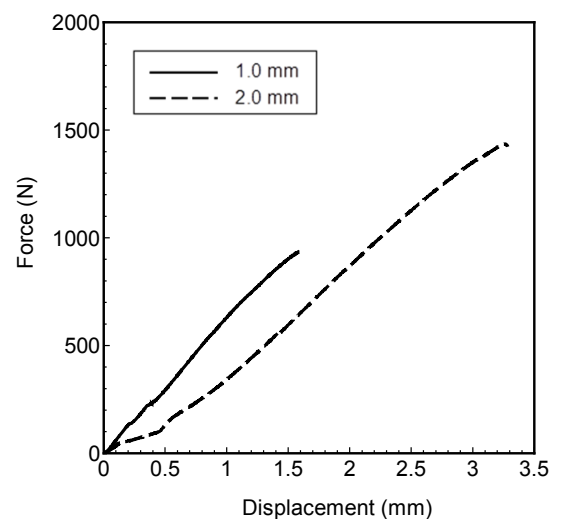

Figure 4: Load-displacement curves for T shape tensile tests. 


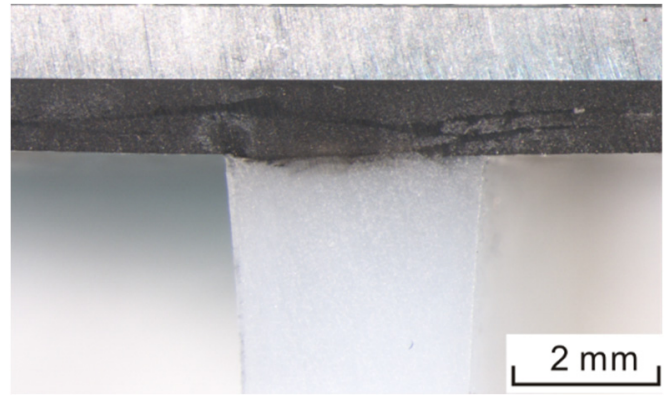

(a) $1.0 \mathrm{~mm}$ thickness

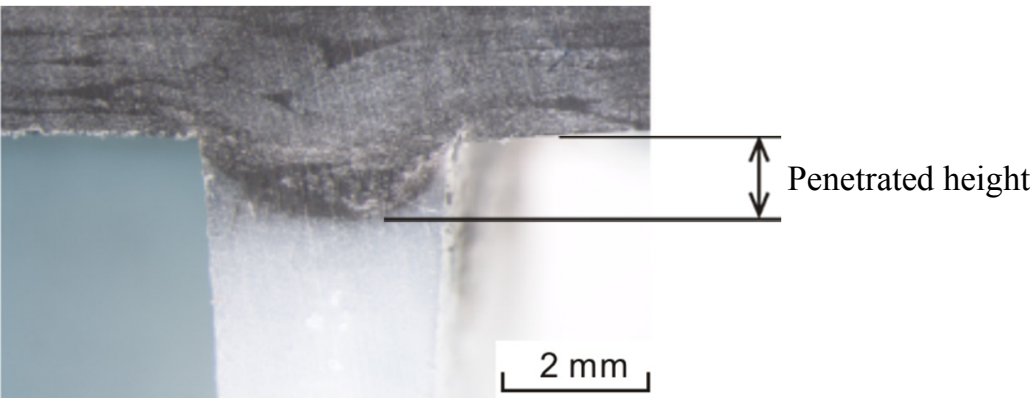

(b) $2.0 \mathrm{~mm}$ thickness

Figure 5: Magnified cross-sectional view of $\mathrm{T}$ shape tensile test specimen.

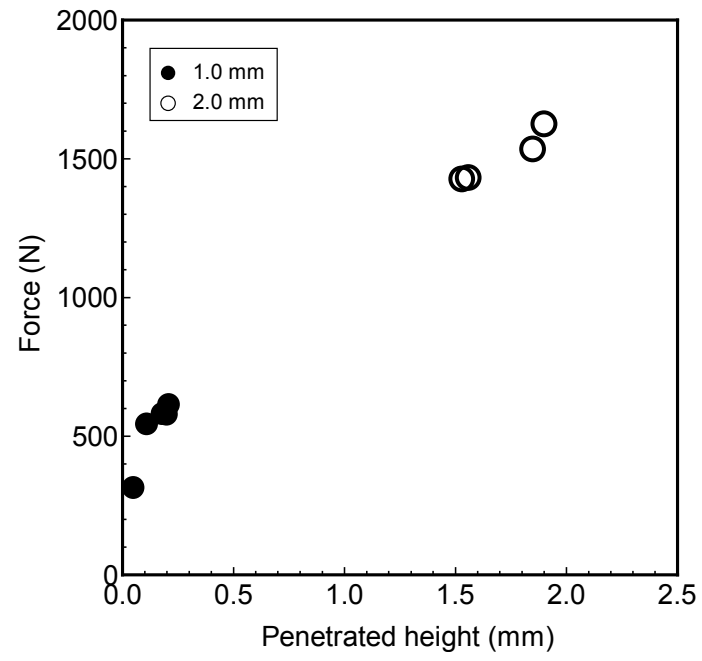

Figure 6: Relationship between penetrated height of laminated outer shell to the rib and fracture force. 
Fig. 7 shows the temperature histories of the surface which will be attached to the injected rib after taking out from the heater. Though the temperature of $1.0 \mathrm{~mm}$ thick laminate suddenly decreased, the temperature of $2.0 \mathrm{~mm}$ thick laminate slowly decreased. As the heat capacity of $2.0 \mathrm{~mm}$ thick laminate is large, the temperature slowly decreased and laminate outer shell had the formability to penetrate into the rib structures during the molding process. This is considered to be the reason for the stronger bonding strength between the injection molded rib structures and the laminated outer shell for the specimen with $2.0 \mathrm{~mm}$ thick laminate.

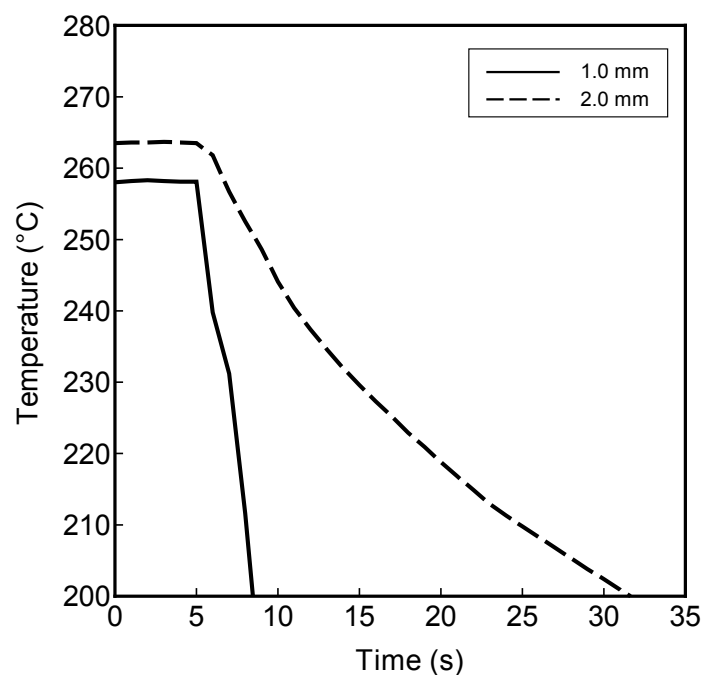

Figure 7: Temperature history of laminated outer shell during molding process.

\section{Conclusions}

In this study, press and injection hybrid molding that can mold products of complex shapes was introduced, and demonstrator products were molded. T shape tensile tests were conducted to evaluate the tensile bonding strength between rib structures and laminated outer shell of the press and injection hybrid molding GFRTP. The investigation yields the following conclusions.

1. The demonstrator products of GFRTP were successfully manufactured by press and injection hybrid molding.

2. As the thickness of the laminated outer shell increased, the tensile bonding strength was increased and the penetrated height became higher. Due to the larger heat capacity of thick laminate, the temperature of the laminate outer shell slowly decreased and it was penetrated into the rib structures. 


\section{References}

[1] "Composites penetration growth in Automotive: towards mass production 2010-2020 trends and forecasts" JEC Composites, pp. 13-22, 2011.

[2] S.W. Beckwith, Thermoplastic composites resins matrices, Sampe Journal, Vol. 44, No.1, pp. 70-71, 2008.

[3] K. Tanaka, N. Kohashi, Y. Kinoshita, T. Katayama and K. Uno, "Compression molding of carbon fiber reinforced thermoplastics using nonwoven stitched multi-axial cloth by means of induction heating system", Journal of the Society of Materials Science, Japan, Vol. 58, No. 7, pp. 642648, 2009.

[4] K. Tanaka, Y. Matsuura, R. Harada, T. Katayama, and S. Enoki, FEM analysis of temperature distribution of CFRTP pipe mold with direct resistance heating, WIT Transactions on the Built Environment, Vol. 137, pp. 265-272, 2014.

[5] K. Tanaka, R. Ushiyama, T. Katayama, S. Enoki and H. Sakamoto, Formability evaluation of carbon fiber NCF by a non-contact 3D strain measurement system and the effects of blank folder force on its formability, WIT Transactions on the Built Environment, Vol. 137, pp. 317-326, 2014.

[6] M. Fiorotto and G. Lucchetta, Experimental investigation of a new hybrid molding process to manufacture high-performance composites, International Journal of Material Forming, Vol. 6, No. 1, pp. 179-185, 2013.

[7] J. Aurrekoetxea, G. Castillo, F. Cortes, M. A. Sarrionandia and I. Urrutibeascoa, Failure of multimaterial fusion bonding interface generated during over-injection molding-thermoforming hybrid process, Journal of applied polymer science, Vol. 102, No. 1, pp. 261-265, 2006. 